\title{
Epidemic host community contribution to mosquito-borne disease transmission: Ross River virus
}

\author{
I. S. KOOLHOF* AND S. CARVER \\ School of Biological Sciences, University of Tasmania, Hobart, Tasmania, Australia
}

Received 2 August 2016; Final revision 20 September 2016; Accepted 1 November 2016; first published online 28 November 2016

\section{SUMMARY}

Most vector-borne diseases infect multiple host species, but disentangling the relative importance of different host species to transmission can be complex. Here we study how host species' abundance and competence (duration and titre of parasitaemia) influence host importance during epidemic scenarios. We evaluate this theory using Ross River virus (RRV, family Togaviridae, genus Alphavirus), a multi-host mosquito-borne disease with significant human health impacts across Australia and Papua New Guinea. We used host contribution models to find the importance of key hosts (possums, wallabies, kangaroos, horses, humans) in typical mammal communities around five Australian epidemic centres. We found humans and possums contributed most to epidemic RRV transmission, owing to their high abundances, generally followed by macropods. This supports humans as spillover hosts, and that human-mosquito and possum-mosquito transmission is predominant during epidemics. Sensitivity analyses indicate these findings to be robust across epidemic centres. We emphasize the importance of considering abundance and competence in identifying key hosts (during epidemics in this case), and that competence alone is inadequate. Knowledge of host importance in disease transmission may help to equip health agencies to bring about greater effectiveness of disease mitigation strategies.

Key words: Arboviruses, transmission, vector-borne disease, vectors.

\section{INTRODUCTION}

The majority of pathogens, including emerging and resurging infectious diseases of humans, domestic animals and wildlife, infect multiple host species [1, 2]. Among a community of hosts, the contribution of individual species to transmission can vary in a number of important ways, including through variance in host species abundances, susceptibility to infection, and ability to transmit pathogens. Thus, understanding

\footnotetext{
* Author for correspondence: Mr I. S. Koolhoff School of Biological Sciences, Private Bag 55, Hobart, Tasmania 7001, Australia.

(Email: koolhofi@utas.edu.au)
}

how different host species in a community contribute to transmission is a critical frontier for comprehending, predicting and controlling infectious diseases [3-5]. Vector-borne pathogens [e.g. West Nile virus (WNV), avian malaria, Ross River virus (RRV)] commonly reside in a range of host species [6-9]. Catholic feeding vectors transmit pathogens among host species that vary in their relative abundance and competence - the relationship between titre and duration of parasitaemia (parasite in the blood) $[10,11]$. However, the relative importance of these factors is rarely understood, particularly compared to directly transmitted pathogens [12].

In this study, we focus on disentangling the relative importance of host species in vector-borne disease transmission. The importance of vector abundance 
in disease transmission is of course indisputable, but the role of host community composition is often neglected [10]. Vector feeding preference can cause a marked impact on the relative contribution of a host species to transmission, independent of host community structure, but evidence suggests that vector feeding strategies more commonly reflect the available host community [13-15]. Furthermore, there is compelling evidence to suggest the importance of host communities. For example, WNV and RRV differ in their prevalence in relation to variable host communities across similar climates and landscapes [16-18]. Urbanization and agricultural land usage has been seen to lead to positive increases in the prevalence of WNV and are associated with changes in host communities along these gradients [16]. RRV outbreaks in southwestern Australia do not always occur in years with high vector abundance, suggesting an important influence of reservoir host community on transmission dynamics and spillover to humans ([19] and references therein). Thus, the relative abundance and competence of hosts in communities likely plays a critical role in shaping vector-borne disease transmission $[2,11]$. To study 'key hosts' in vector-borne disease transmission we use an important disease of humans which is endemic in Australia and Papua New Guinea, i.e. RRV (family Togaviridae, genus Alphavirus).

RRV is a mosquito-transmissible virus which causes epidemics, resulting in chronic fatigue and polyarthritic-type symptoms [20]. Epidemiologically, RRV is the most important vector-borne disease in Australia with 1451-9551 human clinical notifications per year at an estimated annual cost of $\$ 15$ million $[4,21,22]$. There are three major vectors for RRV across Australia: Culex annulirostris, Aedes vigilax, and Aedes camptorhynchus [4, 9, 23, 24]. Vector feeding patterns are influenced by the availability, accessibility and abundance of host species [25], and vectors are generally thought of as host generalists $[8,26,27]$. A range of vertebrate host species are exposed to RRV, with the most competent species being marsupials $[9,24]$. The primary reservoir host is often assumed to be macropod marsupials, owing to the duration and titre of viraemia, but their relative status among other potential reservoir species (e.g. other marsupial or placental mammal species) remains uncertain $[9,24]$. The relative abundance of potential host species that are fed upon by RRV vectors differs in epidemic areas of Australia; this may have important consequences for transmission [10]. Furthermore, host competence differs markedly between species [4, $6,10,13,28,29]$. To better understand the contribution of host species to RRV transmission, it is important to consider both varying abundances of host species and their varying competencies in the host community.

In this study, we simultaneously take into account both host competence and abundance to investigate the contribution of hosts to potential RRV transmission. We expand on the notion of humans being a spillover host for this virus, and also their role in sustaining epidemics. We (i) model the contribution of key hosts to RRV transmission using host communities that are representative of varying epidemic areas of Australia; (ii) evaluate how changes in host competences and abundance may alter the relative importance/contribution in host species; and (iii) characterize the general relationship host abundance and competence has on host contribution to viral transmission. We show that, in addition to macropod marsupials and humans, brushtail possums (Trichosurus vulpecula) may have a greater role in epidemic RRV transmission than previously recognized. Our findings support humans as a spillover host for RRV transmission, and illustrate the critical, often underappreciated, importance of host abundance in attributing 'key host' status to hosts of this mosquito-borne disease.

\section{METHODS}

\section{Study regions and host communities}

This study investigates areas where RRV is known to cause epidemics in five Australian States/Territories: Queensland, New South Wales, Western Australia, South Australia, and the Northern Territory (Table 1). Focal study areas for each State/Territory are as follows: Queensland-northeast Brisbane in the level 3 statistical local areas [SLAs; Australian Bureau of Statistics (ABS)] of Chermside, Nundah, and Sandgate; New South Wales - Newcastle (SLA level 3); Western Australia-Mandurah, Mandurah South, and Green fields (SLAs level 2); South Australia-Murray Bridge significant urban area (SLA level 2); and Northern Territory - broadly the northern suburbs of Darwin, which includes all SLAs (level 2) north of Darwin city and west of Buffalo Creek, including Lyons.

We used five host species communities which roughly represent those commonly reported in RRV epidemic areas across Australia. Hosts are generically 
Table 1. Host abundance and competence (viraemia and titre) across the five regions in this study

\begin{tabular}{|c|c|c|c|c|c|}
\hline Host species & State & Density $/ \mathrm{km}^{2}$ & Viraemia (days) & Titre & Ref. \\
\hline Eastern grey kangaroo* & QLD & 4.72 & $3 \cdot 4(2 \cdot 3-6)$ & $6 \cdot 1$ & {$[4,29,37]$} \\
\hline Wallaby† & QLD & $10 \cdot 4$ & $3 \cdot 4(1 \cdot 1-5)$ & $7 \cdot 4$ & {$[17,29,38]$} \\
\hline Horse: & QLD & $0 \cdot 5$ & $3(2-5)$ & $6 \cdot 3$ & {$[17,28]$} \\
\hline Possum§ & QLD & $246 \cdot 9$ & $2(1-3)$ & $7 \cdot 5$ & {$[13,39]$} \\
\hline Human & QLD & $1015 \cdot 1$ & $4(1-6)$ & $6 \cdot 3$ & {$[13,40,41]$} \\
\hline Possum§ & NSW & $173 \cdot 1$ & $2(1-3)$ & $7 \cdot 5$ & {$[42,43]$} \\
\hline Eastern grey kangaroo* & NSW & $19 \cdot 05$ & $3 \cdot 4(2 \cdot 3-6)$ & $6 \cdot 1$ & {$[4,29,37]$} \\
\hline Horset: & NSW & $0 \cdot 5$ & $3(2-5)$ & $6 \cdot 3$ & {$[28,44]$} \\
\hline Human & NSW & $224 \cdot 9$ & $4(1-6)$ & $6 \cdot 3$ & {$[13,41,45]$} \\
\hline Western grey kangaroo\| & WA & $21 \cdot 9$ & $3 \cdot 4(2 \cdot 3-6)$ & $6 \cdot 1$ & {$[4,29,39]$} \\
\hline Horse: & WA & $0 \cdot 5$ & $3(2-5)$ & $6 \cdot 3$ & {$[17,28]$} \\
\hline Possum $\S$ & WA & $251 \cdot 8$ & $2(1-3)$ & $7 \cdot 5$ & {$[6,39]$} \\
\hline Human & WA & $923 \cdot 1$ & $4(1-6)$ & $6 \cdot 3$ & {$[13,41,46]$} \\
\hline Red kangaroo & SA & $4 \cdot 31$ & $3 \cdot 4(2 \cdot 3-6)$ & $6 \cdot 1$ & {$[29,47]$} \\
\hline Possum§ & SA & $42 \cdot 8$ & $2(1-3)$ & $7 \cdot 5$ & {$[6,17]$} \\
\hline Horset: & SA & $0 \cdot 5$ & $3(2-5)$ & $6 \cdot 3$ & {$[17,28]$} \\
\hline Human & SA & 171 & $4(1-6)$ & $6 \cdot 3$ & {$[13,41,48]$} \\
\hline Horse: & NT & $0 \cdot 5$ & $3(2-5)$ & $6 \cdot 3$ & {$[17,28]$} \\
\hline Possum§ & NT & $290 \cdot 5$ & $2(1-3)$ & $7 \cdot 5$ & {$[39,42]$} \\
\hline Wallaby\# & NT & 15 & $3 \cdot 4(1 \cdot 1-5)$ & $7 \cdot 4$ & {$[17,29,38]$} \\
\hline Human & NT & 1351 & $4(1-6)$ & $6 \cdot 3$ & {$[13,41]$} \\
\hline
\end{tabular}

Common names, genus and species and reference: * Eastern grey kangaroo (Macropus giganteus); $\dagger$ wallaby (based on Macropus parryi); $\$$ horse (Equus caballus); $\S$ possum (based on Trichosurus vulpecula); $\|$ Western grey kangaroo (Macropus fliginosus); $\uparrow$ red kangaroo (Macropus rufus); \# wallaby (based on Macropus agilis). Densities are representative values (see Methods section) and variance in these values was considered in sensitivity analysis. Viraemia are presented as reported means and their lower and upper values.

humans, horses (Equus caballus - a putative host of RRV), kangaroos (Macropus giganteus, Macropus fuliginosus and Macropus rufus), possums (Trichosurus vulpecula), and wallabies (Macropus parryi and Macropus agilis). We acknowledge that $\mathrm{RRV}$ vectors will also feed on other vertebrate species (particularly domestic and agricultural animals, and birds), but they are omitted from this study owing to their general 'dead-end' statuses, and because their inclusion added little to model outcomes in preliminary analyses [4, 9]. Instead, we focused on the aforementioned five hosts because they are generically characteristic of host communities in epidemic areas of Australia. Host community composition and density specific to each of our five study areas were calculated from a variety of sources, including published literature and government datum sources (see Table 1).

Where possible, species densities are taken from peer-reviewed literature and converted into a density representing the number of an individual species/ $\mathrm{km}^{2}$. For the abundance of those species not found in the literature, approximate densities were calculated using average values from the other States/Territories or the relative proportions of these species $i$ in climatically similar states, denoted as $\theta_{\#_{i}}$ [see Table 1, equation (1)]. In the following, we define the parameters for $\theta_{\#_{i}}: H_{i}$ the target species density; $P_{i}$ a relative proportion and known density in the respective state; $P_{d_{i}}$ the known density of the proportion of a target species within that state.

$\theta_{\#_{i}}=\frac{H_{i}}{P_{i}} \times P_{d_{i}}$.

The human population density element to host communities was derived from the ABS. Possum populations were derived from a general possum density per household ratio from the literature [30], and scaled to human population information gathered from the ABS. From the datum we were able to approximate possum densities around these epidemic areas for each state [equation (2)]. Parameters for possum density $\theta_{p}$ are defined as: $R_{i}$ the density of humans $/ \mathrm{km}^{2} ; K_{i}$ the number of people per household; and $P_{i}$ the amount of possums per household.

$\theta_{p}=\frac{R_{i}}{K_{i}} \times P_{i}$. 


\section{Host competence}

The titre and viraemia of each host were taken from the literature. In instances where data was unable to be sourced from the literature, values were based on taxonomically similar host species with a known titre and viraemia [see equation (1), Table 1] M. fuliginosus and $M$. rufus titre and viraemia were considered to be the same as M. giganteus. Furthermore, $M$. robustus titre and viraemia were calculated as the average between an $M$. giganteus and a $M$. agilis.

\section{Modelling host contribution}

To investigate the links of host competence and host abundance effects on host contribution to viral transmission, we simulated two models which represent host contribution $=$ (models adapted from those developed by Streicker et al. [2]).

First, we modelled host contribution using the host's competence (maximum, mean, and minimum literature reported) and estimated likely density. In the following we define the following symbols: $H_{i}$ is the abundance $\left(\log _{10}\right.$-transformed) of host species $i$ within a given state, $d_{i}$ is the duration of viraemia $\left(\log _{10}\right.$-transformed) of host species $i, t_{i}$ is the titre ( $\log _{10}$-transformed) of host species $i, j$ is the comparison of all other species. The overall contribution of species $i$ to a community is $n_{i}=H_{i} d_{i} t_{i}$. Given that there are five different species $(N)$ per state, the total community contribution can be calculated by the following: $n=\sum_{j=1}^{N} H_{j} d_{j} t_{j}$. Thus an individual's contribution to a community $\left(\pi_{i}\right)$ can be modelled as the following:

$\pi_{i}=\frac{H_{i} d_{i} t_{i}}{\sum_{j=1}^{N} H_{j} d_{j} t_{j}}$.

Second, we modelled the relative abundance ( $\log _{10}$-transformed) of species within a community to find which species are found in higher densities within each States/Territories endemic community [equation (4)]. From this we define the following equation:

$\bar{A}=\frac{\sum_{j} H_{j}}{N}$,

where $\bar{A}$ is the average density of an individual species across the whole community. We used the variable $\bar{A}$ to compare the relative density of each individual host species in order to find if a species was high in abundance within a community, relative to the remaining species [equation (5)].

$\theta_{A}=\frac{H_{i}}{\bar{A}}$

Third, we investigated each species within each community to determine relative competence, finding which species have a relatively high titre and viraemia compared to the average infection contribution of each State/Territory, where $j$ is the comparison of all species. We define a state's average infection status as $\hat{s}$ [equation (6)].

$\hat{s}=\frac{d_{j} t_{j}}{N}$.

The average infection contribution for each State/ Territory was then used to find each species' infection status within the endemic community [equation (7)].

$\theta_{s}=\frac{d_{i} t_{i}}{\hat{s}}$

Each individual's contribution/importance to transmission for each State/Territory was then plotted three-dimensionally to illustrate the difference in animal contribution in relation to their relative abundance and competence within each community. Because individual hosts can show variation in the duration and titre of viraemia, we evaluated sensitivity of model findings to this by assessing host importance also at lowest detectable titre and viraemia and average titre and viraemia. Potential variability in knowledge of host densities could also impact model outcomes. The density of host species we used across sites are representative, and can also vary over time, we conducted additional sensitivity analyses on this parameter to evaluate how changes in host abundance impact the relative contribution of host species to transmission. Host densities were presented on a $\log _{10}$ scale with the original host densities marked on sensitivity analysis plots for reference. This further helps to facilitate additional perspectives of how mosquitoes may interact with the varying relative abundances (and possible spatial structure) of hosts around urban fringes [i.e. humans (and often possums) are at higher densities in urban areas, and horses and macropods are at higher densities in around the fringes of urban areas] within each epidemic community. 

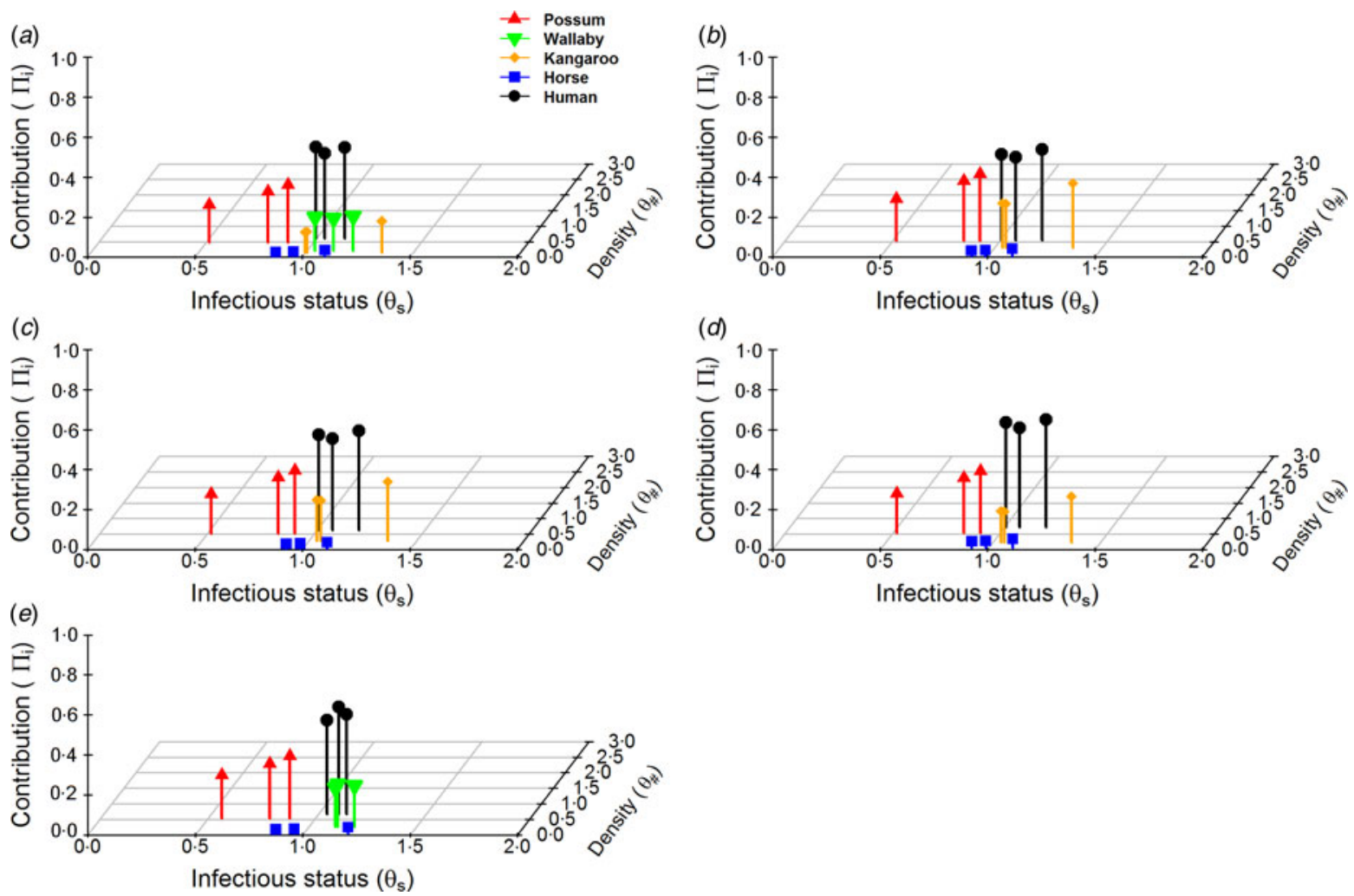

Fig. 1. Host contribution models. The relative contribution/importance of five hosts within Ross River virus epidemic communities: (a) Queensland, (b) New South Wales, (c) Western Australia, (d) South Australia, (e) Northern Territory. Host contribution based on the relationship between relative competence and abundance $\left(\log _{10}\right.$ scale) status.

\section{RESULTS}

\section{Host density and competence}

Host community competence was seen to vary between epidemic centres (Table 1). The densities of possums and human across all epidemic centres were higher than all other species. The densities of kangaroos, horses and wallabies in comparison were smaller, with horses having the lowest estimated densities across all areas. Unsurprisingly, kangaroos were the most competent hosts, mostly owing to their long duration of viraemia (Table 1, Fig. 1). This was followed by humans having a long viraemic period but low titre, wallabies, having both long viraemic periods and high titres, horses with moderate viraemic periods and titre, and then possums, having a short viraemic period, but the highest titre.

\section{Contribution/importance of key hosts to transmission}

Despite variance in host community structure in epidemic regions, we detected reasonable consistency in which hosts were potentially most important for RRV transmission. Possums and humans were the most important hosts for transmission of RRV in each epidemic community (Fig. 1). These hosts were closely followed in importance by kangaroos and wallabies in areas where they were present (Fig. 1). Overall, horses had a much smaller contribution to RRV transmission which was consistently due to their relatively low abundances (Fig. 1). Across all hosts, contribution to RRV transmission was most strongly influenced by the host's relative abundance. Our sensitivity analysis showed that while changes in a host competence does change a host's contribution, its magnitude is minor compared to that of its abundance within a community (Fig. 1).

\section{Sensitivity of host abundance on contribution/ importance to transmission}

Because data on the density of host species across our sites is considered representative, and can also vary over time, we also evaluated the sensitivity of host species $i$ 's abundance ( $\log _{10}$-transformed) on contribution to transmission (Fig. 2). Notably, this gave a measure of the abundance change needed for a given host species $i$ to alter its rank order among the other hosts in the community. 

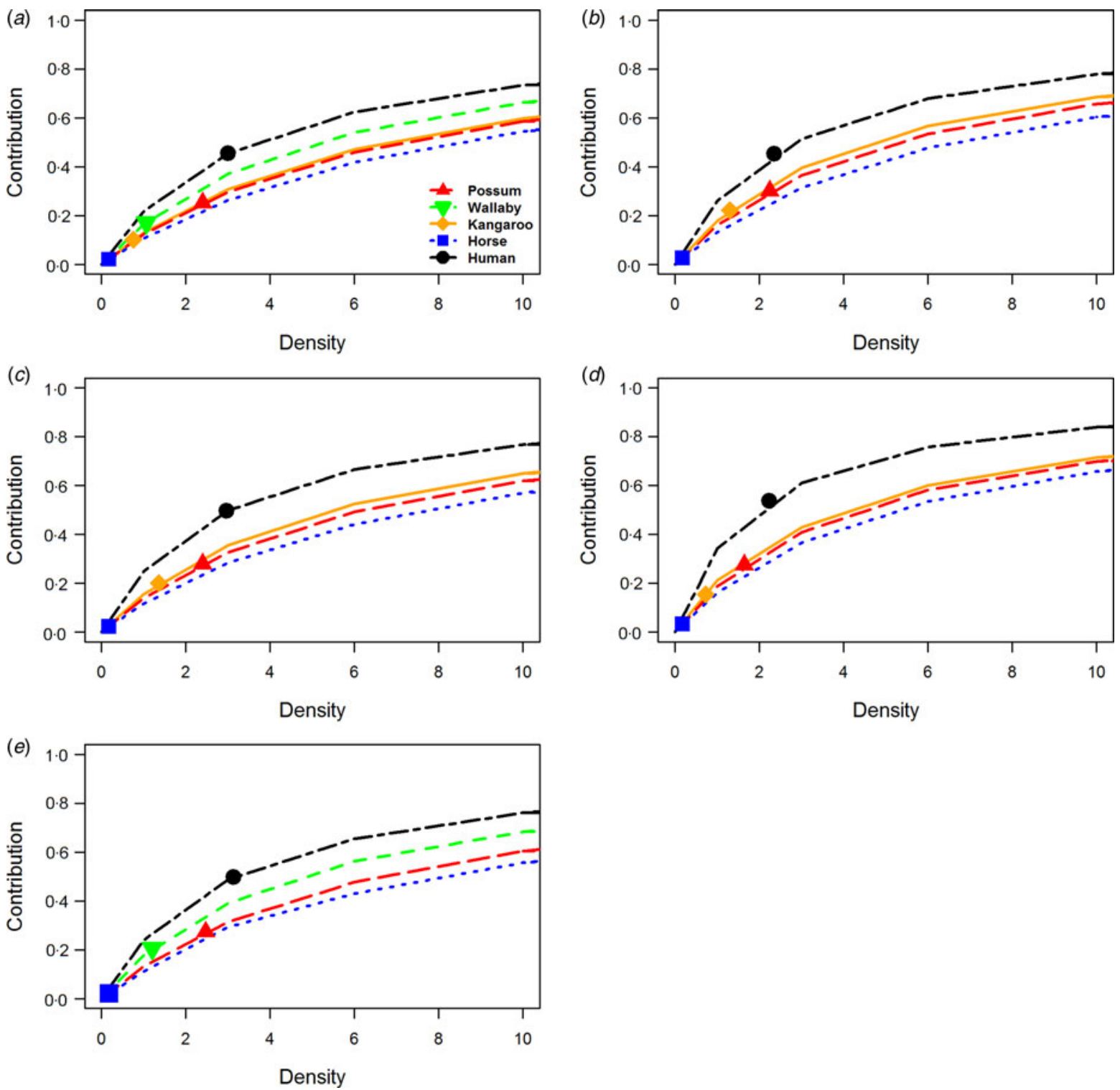

Fig. 2. Host sensitivity models. Sensitivity analysis of the contribution/importance of five host species in epidemic communities: (a) Queensland, (b) New South Wales, (c) Western Australia, (d) South Australia, (e) Northern Territory. Host species change in contribution due to change in $\left(\log _{10}\right.$-scaled) abundance relative to other host species.

Among the sites, possum species densities required more than two orders of magnitude to match human contributions to RRV transmission given the remaining species' densities stayed the same. The remaining species - wallabies, kangaroos, and horses - require vastly unrealistic values up to and exceeding 10 orders of magnitude to contribute similar importance to RRV transmission during epidemics. However, when excluding humans, the host species may compete against each other with only minor increases in their densities needed to overtake one another in their importance to transmission during epidemics. This demonstrates that, even with uncertainty in the absolute densities of hosts, our estimates of the rank order of importance, relative to humans, is robust.

\section{The relationship between host abundance and competence on contribution/importance}

Combining the information among sites and hosts in this study, we determined general relationships between host abundance and competence on contribution to transmission [Fig. 3: contribution $\sim \log _{10}$ (relative abundance) $* 0 \cdot 137$ (s.E. $=0 \cdot 007)+$ competence $* 0.083$ (s.E. $=$ $\left.0 \cdot 20), r^{2}=0 \cdot 875\right]$. Intuitively, the contribution of a host in a community increases with higher abundance 


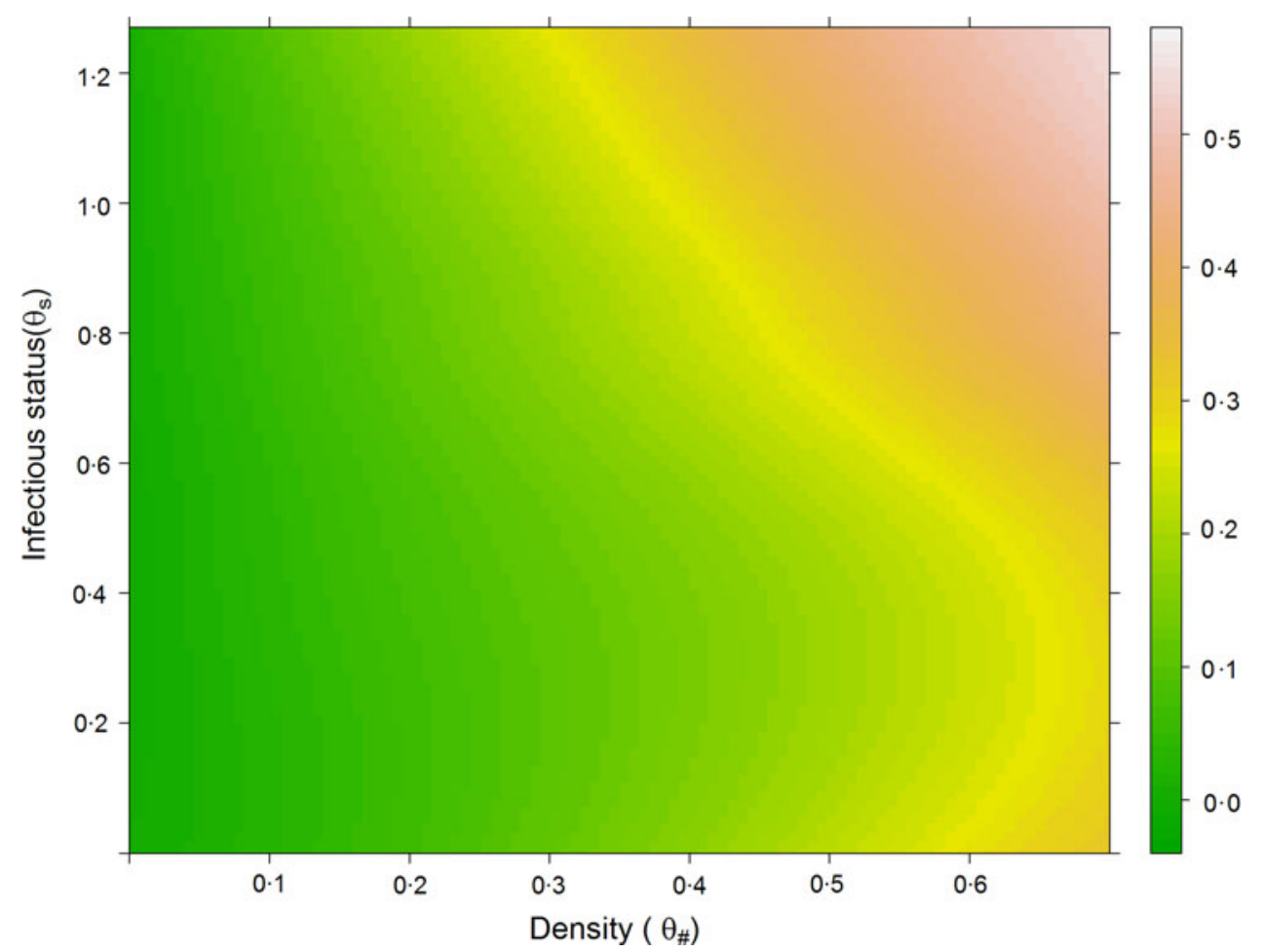

Fig. 3. Contribution association heat map. Combined contribution to transmission of all hosts affected by infectious status (upper, mean, and lower competence) and abundance (density): $\log _{10}$-scaled host densities.

and higher competence. However, it is notable that host abundance appeared to have a more dominant effect on host importance to transmission than that of competence.

\section{DISCUSSION}

In this study, we sought to elucidate the relative importance of host species in a community on mosquito-borne disease transmission-focusing on RRV in epidemic centres of Australia. We considered the epidemiologically important parameters of host competence and abundance $[2,11]$, assuming catholic feeding vector(s). RRV transmission is regulated by interactions between vectors, reservoir host competence, and the relative abundance of hosts within the communities, and this varies across Australia [4, 9]. We found that within communities human and possum hosts have the greatest potential to contribute to viral transmission during epidemics based on their relative competence and abundance, regardless of geographical location. Despite having the greatest competence, the lowest abundance of kangaroos resulted in their lower potential contribution to epidemic transmission, compared to human and possum hosts. Wallabies had broadly similar potential to contribute to RRV transmission as kangaroos, and horses were consistently minor contributors to transmission owing to their low abundance within the communities. Thus, these results indicate that within epidemic areas, macropods may play a lesser role as a 'key host' for RRV during epidemics. Instead, transmission during epidemics in these areas may be dominated by mosquito-human and mosquito-possum interactions. Furthermore, we show that when considering a host's infectious status and competence, high host abundance can swamp the relative contribution of a host species to RRV transmission during an epidemic.

While our results suggests that humans and possums are potential 'key hosts' for RRV in epidemic centres, we also acknowledge that humans do not sustain RRV transmission [10, 31]. Indeed evidence suggests there can be short-lived human-mosquitohuman transmission cycles, but these do not persist to become endemic [8]. Important distinctions here are that of endemic and epidemic host communities, as well as 'key hosts' vs. sustained transmission. We focussed on epidemic centres of RRV transmission owing to the epidemiological importance, and also the greater availability of host and disease information, relative to RRV transmission in non-epidemic areas. Our modelling contributes new insights into 
the 'key hosts' in a community during epidemics of mosquito-borne disease, but this should not be confused with endemic transmission, which is associated with persistent transmission cycles. If anything, the disparity between what are generally considered the putative reservoir hosts of RRV (macropods), and the 'key hosts' in epidemic communities identified here (humans and possums), may suggest that endemic RRV transmission is maintained at larger scales, which are not yet well understood.

Representing the contribution of host species in differing scenarios (changes in abundance and competence) has enabled us to keep the rank-order of host abundance the same, but facilitate additional perspectives of how mosquitoes may interact with the varying relative abundances (and possible spatial structure) of hosts within each epidemic community. For example, humans (and often possums) are at higher densities in urban areas than macropods. Similarly, horses and macropods are at higher densities around the fringes of urban areas than humans. Ultimately, we do not know the functional impact of a host's spatial distribution around these epidemic areas on relative host importance to RRV transmission. Our models suggest that human, possum, and kangaroo hosts require the least change in abundance to markedly alter their contribution to viral transmission. We suspect host contributions may vary over time based on fluctuations in vector and hosts abundances. Additionally, herd immunity in hosts likely plays a role in transmission and spillover occurrence into human populations $[10,32]$.

Herd immunity was not considered in the models presented here. However, herd immunity plays a vital role in the number of competent hosts within communities capable of supplying vectors with a viable bloodmeal needed for viral transmission [19]. In particular, potential reservoir hosts differ in lifespan and reproductive output. This translates into difference in the emergence of new available competent hosts entering epidemic host communities through time and difference among host reservoir immunities [10]. The role of herd immunity is more apparent in viral transmission and host contribution when considering the host's spatial location and density to humans. For instance, while humans may be in closer spatial proximity to horses in suburban areas, the relative long lifespan of horses may mean that their herd immunity remains at a higher level, relative to hosts with shorter lifespan, thus limiting their role in RRV transmission into human populations. In contrast, macropod marsupials, while living further from suburban areas, live relatively shorter lives than horses and breed at younger ages. This allows for their offspring to enter epidemic host communities as susceptible hosts capable of viral transmission. While our models do not explicitly capture these aspects of herd immunity, our results nevertheless still show horses playing a very minor role in epidemic transmission, albeit through the mechanism of low abundance. It is therefore possible that the importance of horses in RRV transmission may be even less than our models predict.

An often discussed limitation to comprehending the role of non-human hosts in the transmission of RRV is a lack of knowledge regarding their abundance, both at spatial and temporal scales [19,33]. This limitation has been acknowledged for decades $[4,8,9,17$, 19]. For example, the lack of literature surrounding rodents' spatial distributions and abundances led to their omission from this study, in spite of their potential ability to contribute to RRV transmission [34]. For this study we targeted the knowledge gap of host contribution and have (at least partially) overcome this limitation. Here we made the assumption that there was random mixing of mosquitoes among host species with mosquitoes being host generalists feeding on all hosts according to their relative abundance. To this end, we have made substantive efforts to identify representative densities of likely RRV hosts around epidemic areas of Australia, and accounted for uncertainty in their estimates through our sensitivity analyses. Furthermore, modelling host competence across the range host viral activity seen in each respective animal has enabled us to quantify the 'key hosts' during RRV epidemics. This highlights the importance of a host's abundance within a community and its contribution to transmission during epidemics. Such insights have mostly been speculative in the past. We hope that the information we have generated on host abundances among some of Australia's most important epidemic locations will contribute positively to further research that targets the gaps in host ecology with regard to the epidemiology of this disease.

We believe the 'key host' role we identified for humans in RRV transmission is analogous to an amplification effect [12], following viral spillover events. Human densities are markedly higher than those of other hosts, and while human hosts retain a low titre, their viraemia duration is long [13] relative to other hosts in our study communities. Consequently, our interpretations of relative 
host importance are likely robust. These results demonstrate that changes in host abundances needed for other host species to become as important in epidemic RRV transmission are mostly unrealistic. It is also important to acknowledge perceptions of the RRV reservoir host as macropods; predominantly due to their high competence $[9,35]$. Our results suggest macropods potentially play a lesser role during epidemics, and instead (in contrast to the literature) possums may have a greatly enhanced contribution, owing to their competence, high abundance and urban associations with humans. This result is supported by other modelling studies [14]. This of course does not suggest that macropods are not reservoirs of RRV transmission, but that their role may be more focused around enzootic and epizootic phases of transmission, than epidemic.

This study provides valuable insight on the interactions between host competence and host abundance in epidemic communities. However, results from this study do have limitations. Vector characteristics were not accounted for in our models and were beyond the scope of this study. We assumed that there was no vector feeding preference across all hosts. While available evidence suggests the dominant RRV vectors are catholic (at least within mammals), some host preferences may exist [26, 36]. Empirical and theoretical studies on vector feeding preferences would be a valuable extension to understanding RRV transmission in epidemic communities. Furthermore, our models did not consider vector abundance or changes in seasonal conditions, nor exposure times, as human hosts are not as frequently in contact with vectors compared to other host counterparts. Potentially, this may suggest possums have an even greater relative role in the epidemiology of RRV than indicated here. Host interaction and mixing was also assumed to be random and constant on both a spatial and temporal scale. Although these factors may alter some aspects of the host contribution, it was first vital to find the effect species abundance and competence has on viral transmission. From our models, further analysis can be performed using these suggested variables.

\section{CONCLUSIONS}

Disease transmission is a dynamic interplay involving a network of hosts varying in abundance and competence [12]. In this study we set out to disentangle host importance within typical epidemic host communities in the transmission of RRV in specific population centres around Australia that are also applicable to other national areas. For diseases that have the ability to spillover into human populations, determining key hosts in the transmission cycle is vital in mitigating epidemics [31]. In the transmission of RRV, host abundance and competence are key indicators of the relative contributions hosts have in transmission within an epidemic community. From our findings, we enhance understanding of the transmission of vector-borne diseases and the importance of considering both host competence and their relative abundance within epidemic communities. Our results support the notion that during a spillover event of RRV into human populations, humans are able to maintain viral transmission [15]. At least for short periods, our study suggests macropod marsupials have a lesser role in epidemic transmission than previously thought, while possums superseded the role of macropods in transmission during epidemics. Furthermore, we document that changes in host competence is not as important to RRV transmission as that of host abundance during epidemics. Relative host importance to RRV transmission may in the future help to equip health agencies with more in-depth knowledge to bring about greater effectiveness in mitigation strategies aimed at reducing human RRV cases. This potentially includes host immunization, if a vaccine is ever made widely available [21]. For hosts which are long-lived and have long reproductive outputs, such as horses, immunization may form a valuable aspect in reducing the amount of competent hosts available for transmission. For hosts which are shortlived and have higher reproductive turnover, herd immunity through vaccination would prove to be a more challenging undertaking for effective control. Further research into epidemic communities may also look at vector feeding preferences, more detailed host communities, and variance in host exposure to vectors.

\section{ACKNOWLEDGEMENTS}

This research was partially supported by funding from the Western Australia Government Funding Initiative for Mosquito Management in Western Australia, and the UTAS Research Enhancements Grant Scheme to Scott Carver.

\section{DECLARATION OF INTEREST}

None. 


\section{REFERENCES}

1. Jones KE, et al. Global trends in emerging infectious diseases. Nature 2008; 451: 990-993.

2. Streicker DG, Fenton A, Pedersen AB. Differential sources of host species heterogeneity influence the transmission and control of multihost parasites. Ecology Letters 2013; 16: 975-984.

3. Becker DJ, Streicker DG, Altizer S. Linking anthropogenic resources to wildlife-pathogen dynamics: a review and meta-analysis. Ecology Letters 2015; 18: 483-495.

4. Harley D, Sleigh A, Ritchie S. Ross River virus transmission, infection, and disease: a cross-disciplinary review. Clinical Microbiology Reviews 2001; 14: 909932.

5. Manore CA, et al. A network-patch methodology for adapting agent-based models for directly transmitted disease to mosquito-borne disease. Journal of Biological Dynamics 2015; 9: 52-72.

6. Boyd A, Kay BH. Solving the urban puzzle of Ross River and Barmah Forest viruses. Arbrovirus Research in Australia 2001; 8: 14-22.

7. Kilpatrick AM, et al. Host heterogeneity dominates West Nile virus transmission. Proceedings of the Royal Society of London, Series B: Biological Sciences 2006; 273: 2327-2333.

8. Lindsay M, et al. Western Australian arbovirus surveillance and research program, Annual Report: 1997-1998. Arbovirus Surveillance and Research Laboratory and the Western Australian Centre for Pathology and Medical Research. Western Australia Government, 1998.

9. Russell RC. Ross River virus: ecology and distribution. Annual Review of Entomology 2002; 47: 1-31.

10. Carver S, et al. Influence of hosts on the ecology of arboviral transmission: potential mechanisms influencing dengue, Murray valley encephalitis, and Ross River virus in Australia. Vector-Borne and Zoonotic Diseases 2009; 9: 51-64.

11. Kilpatrick AM, et al. West Nile virus epidemics in North America are driven by shifts in mosquito feeding behavior. PLoS Biology 2006; 4: 606-610.

12. Keesing F, Holt RD, Ostfeld RS. Effects of species diversity on disease risk. Ecology Letters 2006; 9: 485498.

13. Boyd AM, Kay BH. Assessment of the potential of dogs and cats as urban reservoirs of Ross River and Barmah Forest viruses. Australian Veterinary Journal 2002; 80: 83-86.

14. Choi YH, et al. Modelling the transmission dynamics of Ross River virus in Southwestern Australia. Journal of Mathematics Applied in Medicine and Biology 2002; 19: $61-74$.

15. Zeilinger AR, Daugherty MP. Vector preference and host defense against infection interact to determine disease dynamics. Oikos 2014; 123: 613-622.

16. Bowden SE, Magori K, Drake JM. Regional differences in the association between land cover and West Nile Virus disease incidence in humans in the United States. American Journal of Tropical Medicine and Hygiene 2011; 84: 234-238.
17. Carver S, et al. Dryland salinity and the ecology of Ross River virus: the ecological underpinnings of the potential for transmission. Vector-Borne and Zoonotic Diseases 2009; 9: 611-622.

18. Ezenwa VO, et al. Land cover variation and West Nile virus prevalence: patterns, processes, and implications for disease control. Vector-Borne and Zoonotic Diseases 2007; 7: 173-180.

19. Carver S, et al. Environmental monitoring to enhance comprehension and control of infectious diseases. Journal of Environmental Monitoring. 2010; 12: 2048 2055.

20. Marshall I, Miles J. Ross River virus and epidemic polyarthritis. Current Topics in Vector Research 1984; 2: 31-56.

21. Aaskov J, Fokine A, Liu W. Ross River virus evolution: implications for vaccine development. Future Virology 2012; 7: 173-178.

22. Australia Governmenal Department of Health. Notifications for all diseases by State \& Territory and year 2016 (http://www9.health.gov.au/cda/source/rpt_2_sel. cfm). Accessed 19 September 2016.

23. Ballard JWO, Marshall ID. An investigation of the potential of Aedes-Camptorhynchus (THOM) as a vector of Ross River virus. Australian Journal of Experimental Biology and Medical Science 1986; 64: 197-200.

24. Monath TP. The Arboviruses: Epidemiology and Ecology. CRC Press Inc., 1988.

25. Kay BH, Boreham PFL, Williams GM. Host preferences and feeding patterns of mosquitos (Diptera, Culicidae) at Kowanyama, Cape York Peninsula, Northern Queensland. Bulletin of Entomological Research 1979; 69: 441-457.

26. Johansen CA, Power SL, Broom AK. Determination of mosquito (Diptera: Culicidae) bloodmeal sources in Western Australia: implications for arbovirus transmission. Journal of Medical Entomology 2009; 46: 11671175 .

27. Lee DJ, Clinton KJ, Ogower AK. The blood sources of some Australian mosquitoes. Australian Journal of Biological Sciences 1954; 7: 282-301.

28. Kay BH, et al. The experimental infection of horses with Murray Valley encephalitis and Ross River viruses. Australian Veterinary Journal 1987; 64: 52-55.

29. Kay B, et al. Experimental infection of vertebrates with Murray Valley encephalitis and Ross River viruses. Arbovirus Research in Australia, Proceedings Symposium, 1986.

30. Kay BH, et al. Mosquito feeding patterns and natural infection of vertebrates with Ross River and Barmah Forest viruses in Brisbane, Australia. American Journal of Tropical Medicine and Hygiene 2007; 76: 417-423.

31. Lindsay M, et al. Ross River and Barmah Forest viruses in Western Australia, 2000/01-2003/04: contrasting patterns of disease activity. Arbovirus Research in Australia 2005; 9: 194-201.

32. Potter A, et al. The seroprevalence and factors associated with Ross River virus infection in Western grey 
kangaroos (Macropus fuliginosus) in Western Australia. Vector-Borne and Zoonotic Diseases 2014; 14: 740-745.

33. Kutt AS, et al. Variation in terrestrial mammal abundance on pastoral and conservation land tenures in north-eastern Australian tropical savannas. Animal Conservation 2012; 15: 416-426.

34. Carver S, et al. House mouse abundance and Ross River virus notifications in Victoria, Australia. International Journal of Infectious Diseases 2008; 12: 528-533.

35. Kay B, Aaskov J. Ross River virus (epidemic polyarthritis). The Arboviruses: Epidemiology and Ecology 1989; 4: 93-112.

36. Apperson CS, et al. Host feeding patterns of established and potential mosquito vectors of West Nile virus in the eastern United States. Vector-Borne and Zoonotic Diseases 2004; 4: 71-82.

37. Southwell CJ, Cairns SC. Abundance of large macropods in the eastern highlands of Australia. Wildlife Society Bulletin 1997; 25: 125-132.

38. Southwell C, et al. Abundance and harvest rate of the whiptail wallaby in southeastern Queensland, Australia. Wildlife Society Bulletin 1995; 23: 726-732.

39. Carver S. Resistance of mammal assemblage structure to dryland salinity in a fragmented landscape. Journal of the Royal Society of Western Australia 2010; 93: 119-128.

40. Australia Bureau of Statistics. National Regional Profile: Queensland Australian Government (http://www.abs. gov.au/AUSSTATS/abs@nrp.nsf/Latestproducts/LGA3 Population/People12007-2011?opendocument\&tabname= Summary\&prodno $=$ LGA3\&issue $=2007-2011$ ). Accessed 4 April 2014.

41. Rosen L, Gubler DJ, Bennett PH. Epidemic polyarthritis (Ross River) virus infection in the Cook Islands. American Journal of Tropical Medicine and Hygiene 1981; 30: 1294-1302.
42. Boyd AM, et al. Experimental infection of Australian brushtail possums, Trichosurus vulpecula (Phalangeridae: Marsupialia), with Ross River and Barmah Forest viruses by use of a natural mosquito vector system. American Journal of Tropical Medicine and Hygiene 2001; 65: 777-782.

43. Kerle JA, Kimmorley C, Old JM. An inland population of the common ringtail possum (Pseudocheirus peregrinus) at Black Rock Ridge, New South Wales: a preliminary ecological assessment. Australian Mammalogy 2013; 35: 236-240.

44. Vernes K, Freeman M, Nesbitt B. Estimating the density of free-ranging wild horses in rugged gorges using a photographic mark-recapture technique. Wildlife Research 2009; 36: 361-367.

45. Australia Bureau of Statistics. National Regional Profile: New South Wales Australian Government (http://www. abs.gov.au/AUSSTATS/abs@nrp.nsf/Latestproducts/ 1Population/People12007-2011?opendocument\&tabname= Summary\&prodno=1\&issue $=2007-2011$ ). Accessed 4 April 2014.

46. Australia Bureau of Statistics. National Regional Profile: Western Australia: Australian Government (http://www. abs.gov.au/AUSSTATS/abs@nrp.nsf/Latestproducts/ LGA5Population/People12007-2011?opendocument\& tabname $=$ Summary \&prodno $=$ LGA5\&issue $=2007-2011$ ). Accessed 4 April 2014.

47. Cairns SC, Grigg GC. Population-dynamics of red kangaroos (Macropus rufus) in relation to rainfall in the South Australian pastoral zone. Journal of Applied Ecology 1993; 30: 444-458.

48. Australia Bureau of Statistics. National Regional Profile: South Australia: Australian Government (http://www. abs.gov.au/AUSSTATS/abs@nrp.nsf/Latestproducts/ LGA4Population/People12007-2011?opendocument\& tabname $=$ Summary \&prodno $=$ LGA4\&issue $=2007-2011$.). Accessed 4 April 2014. 